\title{
Illusory Movement of Stationary Stimuli in the Visual Periphery: Evidence for a Strong Centrifugal Prior in Motion Processing
}

\author{
Ruyuan Zhang, ${ }^{1 \star}$ Oh-Sang Kwon, ${ }^{1 *}$ and Duje Tadin ${ }^{1,2}$ \\ ${ }^{1}$ Department of Brain and Cognitive Sciences, Center for Visual Science, and ${ }^{2}$ Department of Ophthalmology, University of Rochester, Rochester, \\ New York 14627
}

Visual input is remarkably diverse. Certain sensory inputs are more probable than others, mirroring statistical regularities of the visual environment. The visual system exploits many of these regularities, resulting, on average, in better inferences about visual stimuli. However, by incorporating prior knowledge into perceptual decisions, visual processing can also result in perceptions that do not match sensory inputs. Such perceptual biases can often reveal unique insights into underlying mechanisms and computations. For example, a prior assumption that objects move slowly can explain a wide range of motion phenomena. The prior on slow speed is usually rationalized by its match with visual input, which typically includes stationary or slow moving objects. However, this only holds for foveal and parafoveal stimulation. The visual periphery tends to be exposed to faster motions, which are biased toward centrifugal directions. Thus, if prior assumptions derive from experience, peripheral motion processing should be biased toward centrifugal speeds. Here, in experiments with human participants, we support this hypothesis and report a novel visual illusion where stationary objects in the visual periphery are perceived as moving centrifugally, while objects moving as fast as $7 \%$ s toward fovea are perceived as stationary. These behavioral results were quantitatively explained by a Bayesian observer that has a strong centrifugal prior. This prior is consistent with both the prevalence of centrifugal motions in the visual periphery and a centrifugal bias of direction tuning in cortical area MT, supporting the notion that visual processing mirrors its input statistics.

\section{Introduction}

The fundamental function of perceptual systems is to provide useful, though not necessarily accurate, information about the environment. The ensuing perceptual experiences abound with occasions where perception does not match sensory inputs (Kersten et al., 1996; Adams et al., 2004). While these perceptual "errors" may seem maladaptive, they often reflect sensory processes that take into account not only sensory inputs but also our prior perceptual experiences, offering unique insights into underlying perceptual mechanisms and computations (Kersten et al., 2004; Knill and Pouget, 2004; Geisler, 2008). This approach has been successfully applied to visual motion perception, which often exhibits systematic and sometimes pronounced misperceptions. These misperceptions can be explained in a Bayesian framework that explicitly incorporates prior knowledge into per-

\footnotetext{
Received Oct. 3, 2012; revised Jan. 4, 2013; accepted Jan. 11, 2013

Author contributions: R.Z., 0.-S.K., and D.T. designed research; R.Z., 0.-S.K., and D.T. performed research; R.Z., 0.-S.K., and D.T. analyzed data; R.Z., 0.-S.K., and D.T. wrote the paper.

This work was supported by National Eye Institute Grant R01 EY019295 to D.T. and National Institutes of Health Grant P30 EY001319. We thank Davis Glasser and Michael Melnick for help with eye tracking. The content is solely the responsibility of the authors and does not necessarily represent the official views of the National Institutes of Health. The authors declare no competing financial interests.

${ }^{*}$ R.Z. and 0.-S.K. contributed equally to this work.

Correspondence should be addressed to Duje Tadin, 317 Meliora Hall, Department of Brain and Cognitive Sciences, University of Rochester, Rochester, NY 14627. E-mail: duje@cvs.rochester.edu.

DOI:10.1523/JNEUROSCI.4744-12.2013

Copyright $\odot 2013$ the authors $\quad 0270-6474 / 13 / 334415-09 \$ 15.00 / 0$
}

ceptual decisions. Specifically, a "slow speed prior" — an assumption that an object is more likely to be static than to move fastcan account for many motion phenomena (Weiss et al., 2002; Stocker and Simoncelli, 2006; Hedges et al., 2011). For example, a decrease in apparent speed at low contrast (Thompson, 1982) is predicted by high uncertainty about stimulus speed at low contrast, which increases the influence of the slow speed prior on motion perception (Stocker and Simoncelli, 2006).

Why is there a prior on slow speed? In general, priors are believed to come from visual experiences (Adams et al., 2004, Sotiropoulos et al., 2011). Indeed, fast-moving objects are relatively rare, as the reader can likely ascertain by examining his/her own environment. Even if the observer is moving, foveal/parafoveal motion signals are slow. The same holds when we are visually pursuing a moving object. However, this is only true for foveal/ parafoveal stimuli. When we are in motion (Warren et al., 2001) or tracking a moving object (Kowler, 2011), the visual periphery is exposed to motion. Moreover, because the optic flow is predominantly expanding, our peripheral experience is biased toward centrifugal motions. This environmental regularity is also evident in the centrifugal bias of direction tuning in cortical area MT (Albright, 1989). Thus, if prior assumptions derive from experiences (Dakin et al., 2005; Girshick et al., 2011), the slow speed prior should not generalize to the visual periphery. Instead, the prior should shift toward centrifugal speeds. Consequently, we should be biased toward perceiving peripheral objects as moving away from fovea. Because the hypothesized prior is asymmetric in 
velocity space, it should also affect stationary stimuli. Specifically, it predicts a novel illusion where stationary stimuli are perceived as moving centrifugally.

To test these predictions, we measured motion perception biases in the visual periphery over a range of stimulus durations. By varying stimulus duration, we manipulated the spread of the temporal frequency spectrum (Fig. 1C; Derrington and Goddard, 1989). This manipulation affected uncertainty about stimulus motion, as confirmed by speed discrimination measurements. From these behavioral data we modeled the shape of the prior distribution, revealing a strong centrifugal prior. This prior can explain observed motion perception biases in the visual periphery, including strong illusory motion that is seen for briefly presented stationary stimuli.

\section{Materials and Methods}

Participants. Five experienced participants (mean age, 28; 5 males) took part in experiments. Two are authors R.Z. and D.T., while the others were naive to the purpose of the study. All had normal or corrected-to-normal vision. All procedures complied with institutional guidelines for human subjects.

Apparatus. Stimuli were created in MATLAB and Psychophysics Toolbox (Brainard, 1997; Pelli, 1997) and presented on a linearized CRT monitor (24 inch Sony GDM-FW900, $1024 \times$ 640 resolution, $120 \mathrm{~Hz}$ ). Viewing was binocular at $83 \mathrm{~cm}$ (yielding 2 arcmin per pixel) and enforced using a chin rest. As this study required relatively large eccentricity $\left(40^{\circ}\right)$, a second display was used to present fixation targets (Fig. 1A; 9 inch LCD, $600 \times 800$ resolution, $75 \mathrm{~Hz}$ ). The ambient luminance was $0.1 \mathrm{~cd} / \mathrm{m}^{2}$. The background (i.e., gray level) luminance of the stimulus and the fixation monitors were 42 and $30 \mathrm{~cd} / \mathrm{m}^{2}$, respectively.

Direction discrimination experiment (Experiment 1). Stimuli were drifting gratings (spatial frequency, 0.375 cycles $^{\circ}{ }^{\circ}$; contrast, $99 \%$; random starting phase) shown at $40^{\circ}$ eccentricity in either the left or right visual fields (separate blocks). Their spatial extent was set by a stationary raised cosine envelope with an $8^{\circ}$ radius. The temporal contrast envelope, a Gaussian cutoff at $\pm 3 \sigma$, was used to control the stimulus duration. Duration was defined as $\sigma$ of the temporal Gaussian. Contrast was defined as the peak contrast of the temporal contrast envelope. Stimulus velocity and duration were varied. Here, we define centrifugal velocities (i.e., movement away from fovea) as negative and centripetal velocities (i.e., movement toward fovea) as positive.

Using the method of constant stimuli, we estimated the stimulus velocity at which these peripherally presented gratings were perceived as stationary. This was done for four different durations $(\sigma=10,20,80$, and $240 \mathrm{~ms}$ ). Participants completed one practice and eight experimental sessions, with all four durations tested in each session (with their order counterbalanced). For each duration block we selected a range of velocities to bracket psychometric functions, collecting 40 experimental trials for each velocity. The results were fit with the cumulative Gaussian functions. Data analysis revealed slightly more variable results in the visual field on the same side as the nondominant eye. Thus, we considered "dominant" and "nondominant" visual fields separately.

Each trial (Fig. $1 B)$ started with a fixation cross $\left(1.3^{\circ}\right.$ by $\left.1.3^{\circ}\right)$. After $1.5 \mathrm{~s}$, a beep signaled the upcoming stimulus onset, which occurred $300-$ $700 \mathrm{~ms}$ later. Upon stimulus offset, participants made a decision about the perceived motion direction (toward fovea vs away from fovea). No feedback was provided. We ran a control condition to test whether eye movements were a factor underlying the observed results. Here, one participant repeated the direction discrimination experiment with a $2^{\circ}$ fixation window that was enforced by an eye tracker (EyeLink 1000, SR Research). The pattern of the results was nearly identical to the data reported in Figure 2. Moreover, the correlation between the nulling speed estimates (Fig. 2B) obtained when eye fixation was enforced by the eye tracker and the data when eye fixation was self-enforced was 0.998 .

Velocity discrimination experiment (Experiment 2). This experiment was conducted to estimate stimulus likelihoods required by the models described below. The likelihood function, an essential component of Bayesian estimation, is assumed to reflect perceptual variability at the measurement encoding stage (Kersten, 1994). To estimate this key aspect of sensory noise for different durations, we measured just noticeable differences (JNDs) in speed across velocity space. Such measurements are considered to reflect the variance of corresponding sensory measurements, with larger JNDs indicating noisier sensory measurements (Ernst et al., 2002).

To measure JNDs for velocity difference, we kept most of the settings that were in the direction discrimination experiment. The key change was that the experimental task was different. Namely, two drifting gratings were presented sequentially and participants were asked to judge which grating was moving faster toward the fovea (e.g., if both gratings appeared to move away from fovea, the participants picked the slower grating). The interstimulus interval (ISI) for different stimulus durations varied between 200 and 550 $\mathrm{ms}$. This was done because of the long tails of Gaussian temporal envelopes (i.e., to avoid subjective impressions of prolonged ISIs for long-duration stimuli). The two stimuli were a standard grating and a comparison grating. Standard stimuli were the same stimuli (in terms of their velocity and dura- 
tion) as those used in the direction discrimination experiment. Comparison stimuli matched standard stimuli except that their velocity was different, with the amount of difference adjusted by pairs of interleaved staircases (three-up/one-down and two-up/one-down; Levitt, 1971). The results were fit with the cumulative Gaussian functions (see Eq. 1 below), whose standard deviations $(\sigma)$ were taken as JNDs $\left(\sigma_{J N D}\right)$.

Four participants from Experiment 1 took part in the velocity discrimination experiment. Only one visual field location was tested for each participant, chosen as $40^{\circ}$ eccentricity on the dominant eye's side. For each duration we estimated JNDs for three to four velocities. Specific velocities were selected with an aim to span the psychometric functions shown in Figure $2 A$. This was done separately for each participant to ensure appropriate coverage of relevant velocities. Each block of trials involved one stimulus duration, with different velocities randomly interleaved. Each duration was tested in two such blocks, with the order of blocks counterbalanced. Overall, JNDs for each duration-velocity combination were based on the results from four staircases. Across participants and conditions, JNDs ranged between $\sim 0.5 \%$ and $\sim 10 \%$, covering comparison stimulus velocities between $-10 \%$ and $26 \%$ s (Note that these velocities refer to the estimated JNDs. The actual range of velocities used to estimate these JNDs was larger.)

Preliminary estimation of $\sigma_{J N D}$ for each duration and velocity revealed that JNDs for a given duration did not show systematic changes over the tested range of velocities (Fig. 3A). Although departing from Weber's law, this result is consistent with prior studies where constant JNDs were found for velocities close to 0\% (McKee, 1981; Orban et al., 1984; De Bruyn and Orban, 1988; Hedges et al., 2011) (note that although some of our velocities were physically fast, they were perceived as near stationary by our subjects; Fig. 2A). Because of this JND constancy, we assumed a constant JND across velocities for each duration in the main analysis.

Likelihood function estimation. Likelihood functions, reflecting measurement of noisy sensory inputs for different stimuli, were modeled as cumulative Gaussian distributions centered at the actual stimulus velocity. First, we directly fitted the results of the velocity discrimination experiment (Experiment 2) with cumulative Gaussian functions:

$$
\operatorname{Pr}\left(v_{1}>v_{2}\right)=\text { lapse } \times \text { bias }+(1-\text { lapse }) \Phi\left(\frac{v_{1}-v_{2}}{\sigma_{J N D}}\right),
$$

where $\nu_{1}$ and $\nu_{2}$ represent velocities of the two comparison stimuli, $\Phi$ is the cumulative standard Gaussian distribution, and $\sigma_{J N D}$ is its standard deviation. The lapse parameter is used to account for a small number of trials where participants made careless errors, while the bias parameter is used to allow asymmetric distribution of lapse decisions (i.e., bias in lapse responses) (Wichmann and Hill, 2001). The mean estimates for lapse rate and bias parameters were 0.060 and 0.51 , indicating an acceptable lapse rate with no systematic bias. These lapse and bias values, estimated by directly fitting the raw data for each participant and each stimulus duration, were used as constants in the main analysis. This allowed us to limit the number of free parameters.

The standard deviation from the velocity discrimination experiment $\left(\sigma_{I N D}\right)$ relates to the internal sensory noise $\left(\sigma_{\text {sense }}\right)$ in the following way:

$$
\sigma_{I N D}=\sqrt{\operatorname{var}\left(v_{1}-v_{2}\right)}=\sqrt{\sigma_{\text {sense }}^{2}+\sigma_{\text {sense }}^{2}}=\sqrt{2} \sigma_{\text {sense }} .
$$

Specifically, $\sigma_{J N D}$ represents the variability of velocity differences between the two comparison stimuli $\left(\nu_{1}, \nu_{2}\right)$ whose individual variability is set by $\sigma_{\text {sense }}$. Thus, the standard deviation of the likelihood function $\left(\sigma_{\text {sense }}\right)$ was defined as $\sigma_{I N D} / \sqrt{2}$. This assumption was confirmed by a control model described at the end of the Materials and Methods section.

Reconstruction of prior distributions. The prior density function was estimated by two approaches. First, we aimed to estimate the prior without making assumptions about the specific shape of its distribution. Here, the prior density function was not restricted to a particular parameterized family. Instead, we searched the function space by assuming that the density function follows the Gaussian process (Leonard, 1978). The Gaussian process provides a straightforward way of specifying the prior probability of nonparametric density functions. Here, we discretized the prior density function at 25 control points $(X=[-16,-14, \ldots, 30$, $32 \% \mathrm{~s}]$ ) spaced fine enough to approximate most continuous functions. We also implemented a control model that included a wider prior domain $(X=[-28,-26, \ldots, 34,36 \%$ s $]$, yielding 33 control points $)$ and found no notable differences in the results. The density values of the control points followed zero mean $n$-dimensional Gaussian distribution with covariance matrix $\Sigma_{p}$, where $n$ is the number of control points:

$$
\operatorname{den}_{G P}(X) \sim N\left(0, \Sigma_{P}\right)
$$

The entries of the covariance matrix were determined by the squared exponential covariance function, which specifies the covariance of two density values of control points $x_{i}$ and $x_{j}$ as:

$$
\operatorname{Cov}\left(\operatorname{den}_{G P}\left(x_{i}\right), \operatorname{den}_{G P}\left(x_{j}\right)\right)=\exp \left(-\frac{1}{2}\left(\frac{x_{i}-x_{j}}{l}\right)^{2}\right),
$$

where $l$ is a constant determining the smoothness of the density function, set to 4 in all analyses (changes in $l$ had minimal effects on the overall shape of the prior).

In the second approach, we parameterized the prior density function with a skewed Gaussian distribution. This function was chosen based on the results obtained from the nonparametric prior described above. The skewed Gaussian distribution is formalized as:

$$
\operatorname{den}_{S G}(x)=\frac{2}{\sigma} \phi\left(\frac{x-\mu}{\sigma}\right) \Phi\left(\alpha\left(\frac{x-\mu}{\sigma}\right)\right),
$$

where $\phi$ is the standard Gaussian distribution, $\Phi$ is the standard cumulative Gaussian distribution, and $\mu, \sigma$, and $\alpha$ are free parameters representing the central tendency, variability, and skewness of the distribution. This function becomes Gaussian when $\alpha$ is zero, right skewed when $\alpha$ is positive, and left skewed when $\alpha$ is negative.

Fitting Bayesian observer models. For all models, the estimate of velocity $(\hat{\nu})$ is a deterministic function of the sensory measurement $\left(v_{m}\right)$, likelihood function, and the prior distribution:

$$
\hat{v}=\frac{1}{\int \phi\left(\frac{x-v_{m}}{\sigma_{\text {sense }}}\right) d e n(x) d x} \int x \phi\left(\frac{x-v_{m}}{\sigma_{\text {sense }}}\right) d e n(x) d x .
$$

where $\phi$ is a standard Gaussian density function. The probability of "toward fovea" response resulting from sensory input $(\nu)$ is then given:

$$
\begin{gathered}
\operatorname{Pr}(\hat{v}>0)=\Phi\left(\frac{v-\text { criterion }}{\sigma_{\text {sense }}}\right), \\
\text { where } \int x \phi\left(\frac{x-\text { criterion }}{\sigma_{\text {sense }}}\right) \operatorname{den}(x) d x=0 .
\end{gathered}
$$

We numerically approximated integrals of Equations 6 and 7 using the trapezoidal rule.

Once the probability of the toward fovea response $\left(\operatorname{Pr}\left(\hat{\nu}_{i j k}>0\right)\right)$ for a participant $i$, a duration $j$, and a velocity $k$ is given, the number of toward fovea responses $N_{T F(i j k)}$ among the total trials of the condition $N_{\text {total }(i j k)}$ follows binomial distribution:

$$
\operatorname{Pr}\left(N_{T F(i j k)}=n_{T F(i j k)}\right)=\operatorname{binomial}\left(n_{T F(i j k)} ; N_{\text {total }(i j k)}, P\left(\hat{v}_{i j k}>0\right)\right),
$$

where $n_{T F(i j k)}$ is the observed number of toward fovea response. The log-likelihood of the entire direction discrimination dataset (Experiment 1) equals the sum of log-likelihoods of all data points:

$$
\begin{aligned}
& \log \left(\operatorname{Pr}\left(\text { data }_{\text {direction }} \mid \text { params }_{\text {direction }}\right)\right) \\
& =\sum_{i \in S} \sum_{i \in D \in V} \sum_{k \in V} \log \left(\operatorname{Pr}\left(N_{T F(i j k)}=n_{T F(i j k)}\right)\right),
\end{aligned}
$$


where $S, D$, and $V$ represent the sets of participants, durations, and velocities, respectively.

Analogously, the log-likelihood of the entire velocity discrimination dataset (Experiment 2) equals the sum of log-likelihoods of individual responses:

$$
\begin{aligned}
& \log \left(\operatorname{Pr}\left(\text { data }_{\text {velocity }} \mid \text { params }_{\text {velocity }}\right)\right) \\
& \quad=\sum_{i \in S} \sum_{j \in D} \sum_{k \in T} \log \left(\operatorname{Pr}\left(R_{i j k}=r_{i j k}\right)\right),
\end{aligned}
$$

where $S, D$, and $T$ represent the sets of participants, durations, and trials (i.e., velocity differences tested on individual trials) and $r_{i j k}$ is the observed response $\left(\nu_{1}\right.$ or $\left.\nu_{2}\right)$.

We fit the direction discrimination data and the velocity discrimination data concurrently by evaluating the sum of log-likelihood for the two datasets (Eq. 9 and Eq. 10) across the combined parameter space. The prior distributions were fit either to the combined dataset (i.e., one prior distribution for all participants) or, in separate models, to the individual data. All the other parameters were fit to the individual data.

Summary of model parameters. Both the parametric prior and nonparametric prior Bayesian observer models had four parameters associated with the slopes of psychometric functions for velocity discrimination (Experiment 2), i.e., with one sensory noise parameter $\left(\sigma_{\text {sense }}\right)$ for each stimulus duration. As described above, these sensory noise terms were assumed to be $\sigma_{I N D} / \sqrt{2}$. To define prior distributions, 25 control points (i.e., parameters) were used for the nonparametric prior, while three parameters defined the skewed Gaussian prior. A large number of parameters were required for the nonparametric before estimate the prior without initial assumptions about its shape. The estimated prior distributions were relatively smooth (Fig. $4 A, B)$, indicating that our use of 25 control points did not result in overfitting.

Markov Chain, Monte Carlo (MCMC) sampling (using a Metropolis-Hastings algorithm) was used to estimate model parameters. We used a million iterations as a burn-in period and thinned the samples by selecting every 1000th sample in the chain. MCMC sampling technique allowed us to accurately estimate parameter credible intervals (CIs; i.e., Bayesian confidence intervals), which was especially informative in interpreting the reliability of the reconstructed prior distribution. For the nonparametric prior, the estimated 95\% CIs (shaded regions in Fig. $4 A, B$ ) reveal that we were able to precisely estimate the prior shape for velocities that were tested in both the direction and the velocity discrimination experiments (between $-2 \%$ and $16 \%$ s). For velocities outside that range, 95\% CIs gradually increased, indicating that the reliability of the reconstructed prior depends on the velocity.

Control model. Here, we examined the assumption that the standard deviation of the likelihood function $\left(\sigma_{\text {sense }}\right)$ is equivalent to $\sigma_{I N D} / \sqrt{2}$ (see Eq. 2). Specifically, we implemented a nonparametric prior model in which $\sigma_{\text {sense }}$ was a linear function of $\sigma_{I N D}$, such that $\sigma_{\text {sense }}=\alpha+\beta \sigma_{I N D}$. The slope $(\alpha)$ and intercept $(\beta)$ were free parameters. The modeled posterior density of parameters $\alpha$ and $\beta$ were -0.29 and 0.81 . Ninety-five percent CIs for $\alpha$ and $\beta$ were $[-1.37,0.79]$ and $[0.13,1.59]$. Notably, $95 \%$ CIs for $\alpha$ and $\beta$ included zero and $1 / \sqrt{2}$, respectively. The reconstructed prior did not show any qualitative differences from that shown in Figure 4. This confirms that the sensory noise estimated from the velocity discrimination task is consistent with the sensory noise assumed by the participants in the direction discrimination task.

\section{Results}

Behavioral results

To investigate motion perception biases in the visual periphery, we measured changes in perceived motion direction as a function of stimulus duration and velocity. The range of velocities spanned both centrifugal and centripetal directions and always included physically stationary stimuli (velocity $=0 \%$ ). The results (Fig. $2 A$ ) revealed pronounced biases in peripheral motion perception. All stationary stimuli, regardless of their duration, were perceived as moving in the centrifugal direction (i.e., away from fovea). The magnitude of this illusion increased with decreasing stimulus duration. Subjectively, this illusion is very compelling. At $40^{\circ}$ eccentricity, briefly presented stationary stimuli appear to unambiguously move away from fovea.

Next, for each duration we estimated the "point of subjective stationarity," taken as the velocity at which psychometric functions crossed 0.5 (i.e., null velocity illustrated by the dashed line in Fig. 2A). All velocity estimates at which stimuli were perceived as stationary were centripetal, with their magnitude increasing with decreasing duration (Fig. $2 B$ ). This relationship was linear in $\log -\log$ space, with slopes near unity $(0.999$ and 1.01 for dominant and nondominant sides, respectively). For $10 \mathrm{~ms}$ stimulus duration, stimuli needed to be moving toward the fovea at $\sim 7 \%$ to be perceived as stationary-revealing a rather dramatic bias in favor of centrifugal motion directions in visual periphery.

As shown in Figure 2, we found stronger centrifugal biases with shorter stimulus durations. This result likely reflects increasing uncertainty about stimulus motion direction with decreasing duration, which, in turn, increases its susceptibility to internal biases. As discussed below, this increase in directional uncertainty is largely due to the broadening of the temporal frequency spectrum with decreasing duration (Fig. 1C; Derrington and Goddard, 1989). To provide a behavioral estimate of changes in stimulus uncertainty, we measured JNDs for velocity discrimination (see Materials and Methods). The results showed that JNDs were strongly affected by stimulus duration (Fig. $3 A$ ), increasing sharply for brief stimuli. The variations in JND were higher across different durations than across velocity within duration. Thus, to get a JND estimate for each stimulus duration we simply averaged JNDs across velocity (see Materials and Methods for further justification of this step). The result (Fig. $3 B$ ) revealed an exponential relationship between the spread of the temporal frequency spectrum for different durations and measured JNDs, showing that stimulus uncertainty is nicely captured by the stimulus spread in the frequency space. The exponential relationship simply indicates that velocity discrimination performance saturates with increasing duration. 


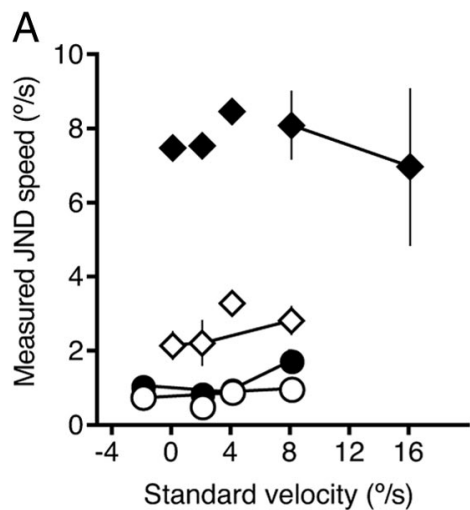

$\mathrm{B}$
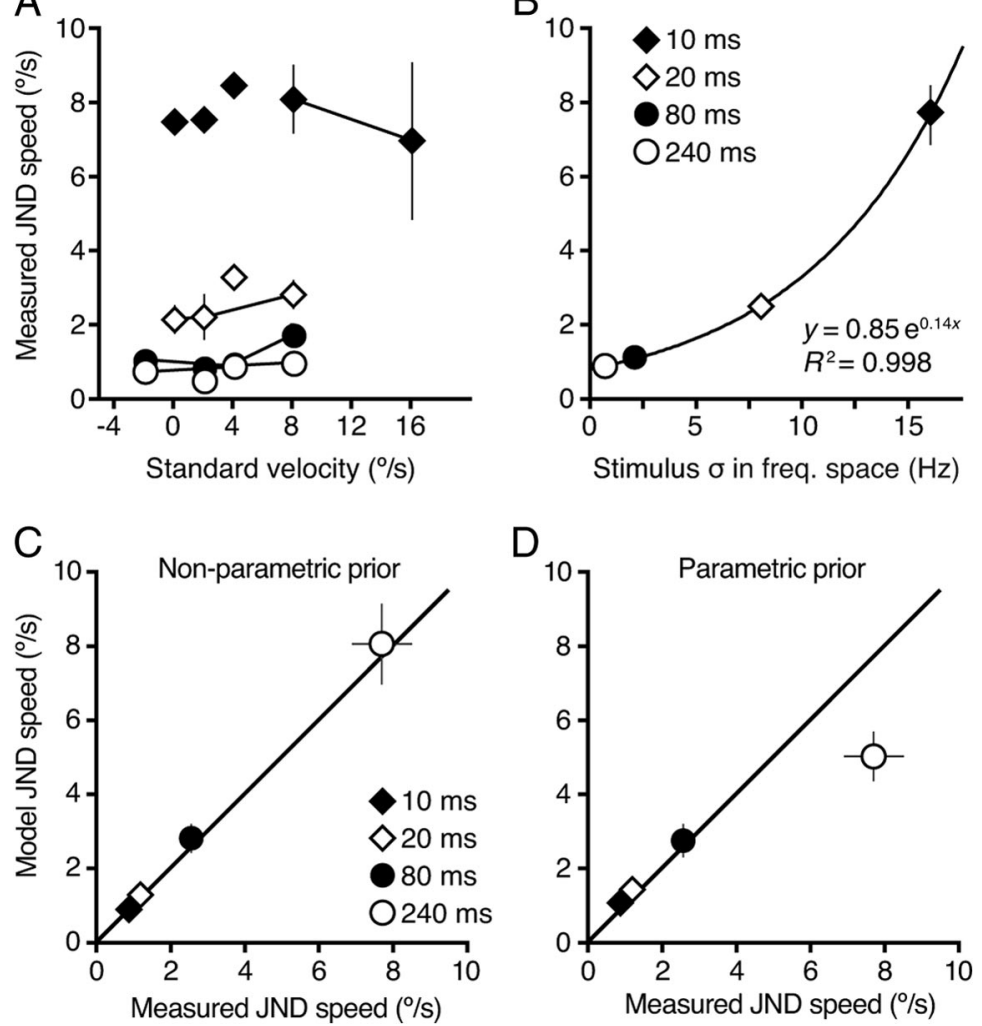

$\mathrm{D}$

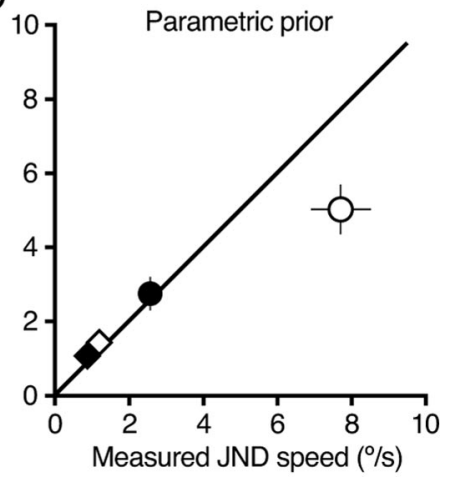

Figure 3. The results and modeling of velocity discriminations. $\boldsymbol{A}$, JNDs were estimated as standard deviations of cumulative Gaussian functions fitted the velocity discrimination data. Different symbols show data for four stimulus durations according to the legend shown in $\boldsymbol{B}$. For each participant, we selected standard velocities to span the dynamic ranges of direction discrimination psychometric functions for each duration (see Fig. 2). This resulted in different velocities tested for different participants. Velocities at which all of the participants were tested are connected with solid lines. $\boldsymbol{B}$, The relationship between stimulus spread in temporal frequency space and the average JND speed for each of four durations. All stimuli were presented in temporal Gaussian envelopes, which yielded corresponding Gaussians in frequency space whose standard deviations indicate temporal frequency spread for different durations. The results were fitted with an exponential function. Error bars are SEM calculated across four participants. $\boldsymbol{C}$, $D$, Model estimated JND speeds (computed as $\sigma_{J N D}=\sqrt{2} \sigma_{\text {sense }}$ ) for the nonparametric prior model $(\boldsymbol{C})$ and the parametric prior model (D). The parametric model underestimates the JND for the shortest duration by $\sim 2.5 \%$. See Results for the implications of this finding.

\section{Bayesian models}

We modeled two Bayesian observers with an aim to explain the observed centrifugal bias by taking into account both durationdependent changes in stimulus uncertainty and a durationindependent prior. Such Bayesian models have proven successful at capturing changes in motion perception resulting from changes in stimulus uncertainty (Hürlimann et al., 2002; Weiss et al., 2002; Stocker and Simoncelli, 2006; Hedges et al., 2011). Here, we describe two similar models that determine motion direction from (1) likelihood function that reflects noise contaminated sensory measurements, and (2) prior assumptions about the peripheral motion signals. Specifically, on each trial Bayesian estimation includes two steps. First, the sensory system measures the physical stimulus speed $\nu$, yielding the measurement $\nu_{m}$, which is variable due to internal sensory noise. Second, the Bayesian observer uses a deterministic function that maps the measured speed $\nu_{m}$ to the estimated speed $\hat{\nu}$. This mapping involves applying Bayes' rule to integrate the likelihood of $\nu_{m}$ given $\nu$ with relevant prior knowledge, resulting in the posterior distribution of velocity. The mean of this posterior distribution determines the model's directional judgment.

The likelihood functions were constrained by both the velocity discrimination experiment (i.e., velocity JNDs) and the direction discrimination task (see Materials and Methods). JND measurements are considered to reflect the variability of corresponding sensory measurements (Ernst and Banks, 2002). Sensory noise also affects direction discrimination judgments (cf. Fig. 2), where it determines the extent to which motion direction judgments are affected by the prior. To examine model performance, we first compared velocity JND estimates obtained by directly fitting the raw data (using Eq. 1) and those estimated by Bayesian models (where $\sigma_{J N D}=\sqrt{2} \sigma_{\text {sense }}$ ). The results show that the sensory noise estimated directly from velocity discriminations is consistent with the modeled sensory noise (Fig. 3C, D). The exception was the $10 \mathrm{~ms}$ duration condition for the model using the parametric prior (Fig. 3D). This and other differences between the models are discussed in a separate section at the end of Results.

Next, we consider the reconstructed prior distributions (Fig. 4). The recovered nonparametric population prior (see $\mathrm{Ma}-$ terials and Methods) is strongly biased toward centrifugal motions (Fig. 4A). The prior has a largely unimodal shape, which is consistent both with priors assumed in earlier studies (Weiss et al., 2002; Sotiropoulos et al., 2011) and with experimentally extracted priors (Stocker and Simoncelli, 2006). However, its peak centers around $8 \%$ in centrifugal direction. This result differs from slow motion priors that were found with foveal/parafoveal stimuli (e.g., Stocker and Simoncelli, 2006), indicating that, for peripherally presented stimuli, human motion processing is biased in favor of centrifugal motion directions. We also observed a consistent but not significant centripetal component (Fig. $4 A$ ). Still, $86 \%$ of the prior distribution covers centrifugal speeds, providing the first computational description of a strong centrifugal bias in peripheral motion processing. In a separate analysis, we also modeled behavioral data assuming independent priors for each participant (Fig. 4B). The centrifugal component of recovered priors was relatively similar across participants. Some differences were found for the centripetal component, where the participant that showed the strongest illusory motion (S2, see open diamonds in Fig. $5 C, D$ ) had the least pronounced centripetal component. This individual difference indicates that between-participant differences in velocity JNDs (i.e., likelihood) cannot fully explain the observed illusory motion perceptions. This is particularly relevant for the briefest stimulus tested $(10 \mathrm{~ms})$, because these were the only stimuli whose likelihood covered fast centripetal speeds (Fig. 3). Namely, for most participants, the strength of the illusory motion for $10 \mathrm{~ms}$ stimuli was not as strong as predicted by velocity JNDs and a strictly centrifugal prior. We will return to this issue in the Model results section below.

Motivated by the recovered nonparametric priors, we ran a separate model where the prior density was parameterized as a skewed Gaussian distribution - a function well suited to capture statistically significant components of the nonparametric prior. By fitting this 
model to the group and individual data, we were able to recover the group (Fig. 4C) and individual priors (Fig. 4D). As with the nonparametric prior, we found that the prior distributions are strongly biased toward centrifugal speeds, with $98.98 \%$ of the prior distribution covering centrifugal speeds. The best fitting parameters for the group data were $\mu=-4.0 \%$ s (95\% CI: -8.7 , $-2.4), \sigma=5.3 \%$ s (95\% CI: $2.8,12.0)$, and $\alpha=-2.1 \%$ s (95\% CI: $-7.1,0.2)$. This nearly significant negative skew indicates a skew toward centrifugal speeds. The mode of the prior density (i.e., its peak), which is different from $\mu$ in skewed Gaussian, was $-7.1 \%$ s (95\% CI: $-9.3,-4.9)$. We found no quantitative or qualitative differences between the group (Fig. 4C) and individual priors (Fig. 4D).

\section{Model results}

Both models closely matched behavioral data, as shown by the fits to the group data (Fig. 5A, B). As duration decreased, both behavioral and model results showed stronger centrifugal biases. Also, stationary stimuli in both sets of results were perceived as moving away from fovea. In sum, the modeled Bayesian observer quantitatively captures psychophysical biases of motion perception in visual periphery. $R^{2}$ values for fits to the individual participants' data using the nonparametric prior ranged between $[0.923,0.976]$ for the group prior, and $[0.976,0.986]$ for individual priors. Individual participant fits using the parametric prior were slightly worse, with $R^{2}$ values between [0.841, 0.971] for the group prior and $[0.954,0.975]$ for individual priors.

We also compared points of subjective stationarity (i.e., the nulling speed) derived from the individual data (Fig. $2 B$ ) with those provided by the models. The results (Fig. $5 C, D$ ) show a close correspondence for both group (solid symbols) and individual (open symbols) data. One slight but systematic error was an overestimation of the nulling speed for $10 \mathrm{~ms}$ stimuli by the parametric prior model (Fig. 5D; points to the right of the dashed line). This illustrates the key difference between the models. By lacking a centripetal component (Fig. 4), the parametric prior model fails to closely fit the data for $10 \mathrm{~ms}$ duration stimuli. This is partially offset by the underestimation of sensory noise $\left(\sigma_{\text {sense }}\right)$ for $10 \mathrm{~ms}$ stimuli by the parametric prior model (Fig. 3D). However, the model still slightly overestimates the magnitude of illusory motion for very brief stimuli. This result suggests three nonexclusive explanations. First, as suggested by the nonparametric prior model, the actual prior might indeed include a centripetal component. As outlined in the Discussion, such prior might have an ecological validity. However, it is also possible that our behavioral measurements either underestimated the magnitude of illusion for $10 \mathrm{~ms}$ stimuli or overestimated velocity JNDs for these brief stimuli.

\section{Discussion}

The diversity in our visual input is astonishing. Certain inputs, however, are more prevalent than others, reflecting the statis-
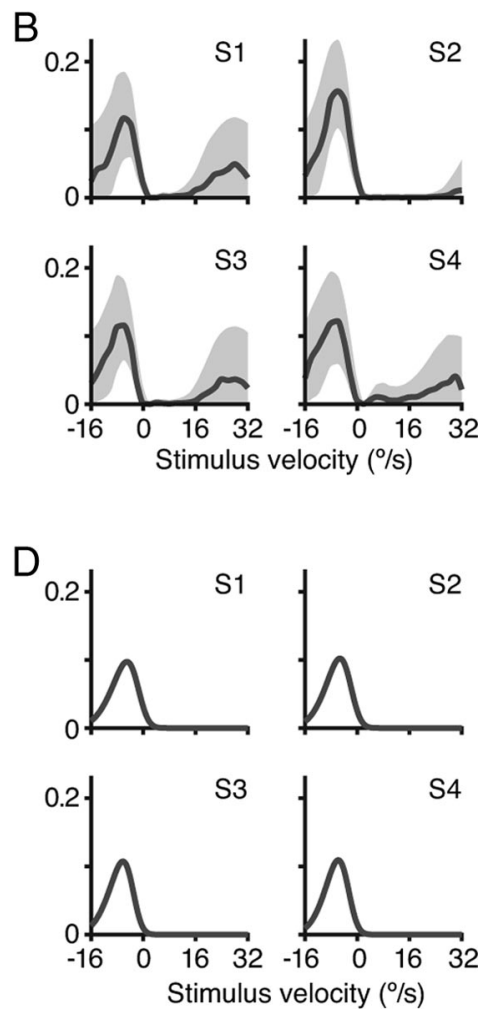

Figure 4. The estimated prior distributions for the nonparametric prior $(\boldsymbol{A}, \boldsymbol{B})$ and the parametric prior $(\boldsymbol{C}, \boldsymbol{D})$ models. $\boldsymbol{A}$ and $\boldsymbol{C}$ show prior distributions fitted to the combined dataset. $\boldsymbol{B}$ and $\boldsymbol{D}$ show prior distributions fitted to the individual data for four

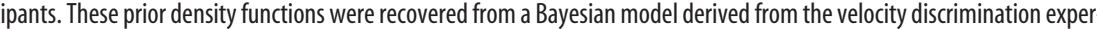

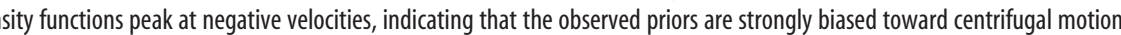
tics of our visual environment (Simoncelli and Olshausen, 2001; Geisler, 2008; Seydell et al., 2010; Girshick et al., 2011). It has long been known that the visual system exploits these statistical regularities, typically resulting in better inferences about our visual environment (Kersten et al., 2004; Geisler, 2008). By making a priori assumptions about incoming visual signals, the overall efficiency of perception improves. However, this comes at the cost of systematic biases (illusions) that often reveal unique insights into underlying mechanisms and computations (Kersten et al., 1996). Here, we report a new visual illusion where stationary objects in the visual periphery are perceived as moving centrifugally, while objects moving as fast as $7 \%$ s are perceived as stationary. These results can be quantitatively explained by a Bayesian prior favoring centrifugal motions in the visual periphery.

While the centrifugal prior revealed here unequivocally differs from previously assumed and/or extracted priors on motion speed and velocity (Hürlimann et al., 2002; Weiss et al., 2002; Stocker and Simoncelli, 2006; Hedges et al., 2011), it is consistent with the Bayesian framework outlined in those studies. Previous studies used foveal/parafoveal stimuli, and the resulting priors appear to qualitatively match input statistics for foveal/parafoveal motions, which are dominated by slow-moving stimuli. The assumption that priors reflect relevant environmental statistics (Girshick et al., 2011) predicts that priors should adjust to changes in input statistics. Indeed, even for centrally presented stimuli, repeated exposure to fast motions can alter the slow speed prior, shifting its mean toward faster speeds (Sotiropoulos et al., 2011). Similarly, sub- 
A
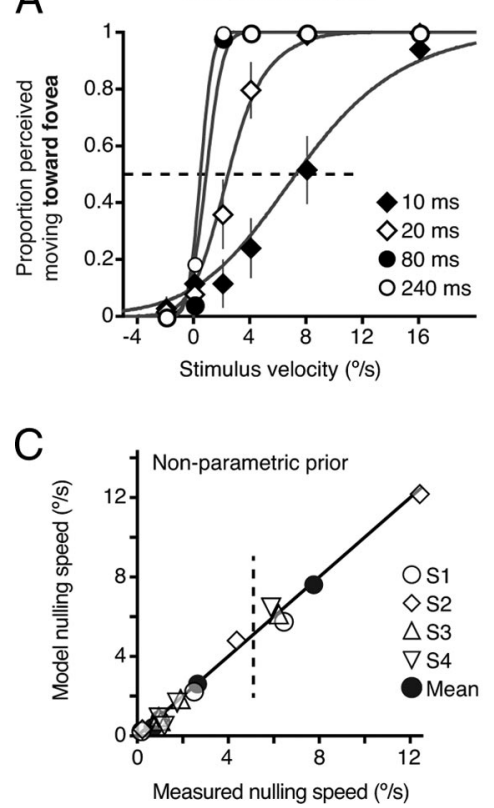

B

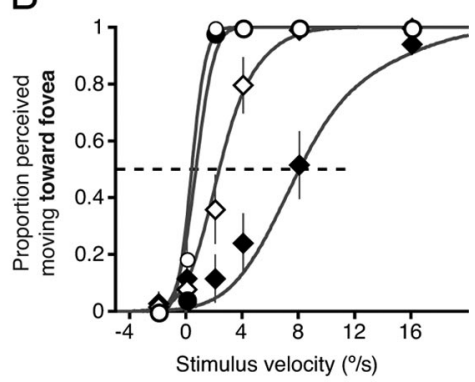

D

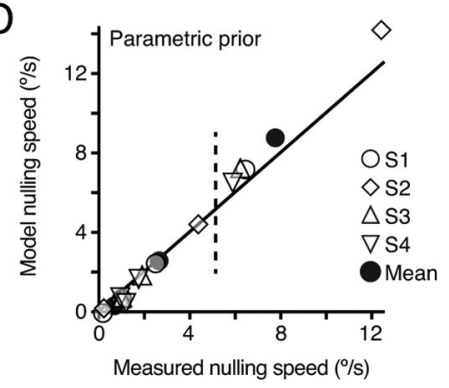

Figure 5. The comparison of modeled and behavioral results. $\boldsymbol{A}, \boldsymbol{B}$, The results are shown for the nonparametric prior $(\boldsymbol{A})$ and parametric prior $(\boldsymbol{B})$ Bayesian observer models. Solid symbols show direction discrimination data for human participants who also completed the velocity discrimination experiments $(n=4)$. The solid lines are the results of the models based on different priors for individual subjects. We found a close correspondence between model and behavioral results $\left(R^{2}=0.989\right.$ and 0.971 for nonparametric and parametric prior models, respectively), with both exhibiting strong centrifugal biases that strengthen with decreasing duration. Models based on unified group priors also provided close fits to the data $\left(R^{2}, 0.991\right.$ and 0.947$)$. Error bars are SEM calculated across four participants. $\boldsymbol{C}, \boldsymbol{D}$, The comparison nulling speeds derived from the raw data (as shown in Fig. $2 B$ ), with nulling speeds provided by the models. Four solid symbols in each panel show average nulling speeds for four different stimulus durations, such that the nulling speed increases with decreasing duration. 0 pen symbols show the same data for individual subjects. The data for the $10 \mathrm{~ms}$ stimulus duration are shown to the right of the dashed lines.

jects that are exposed to a biased distribution of motion directions adjust their expectations accordingly (Chalk et al., 2010). Thus, the empirical evidence indicates that past experience plays a key role in shaping Bayesian priors. The strong centrifugal prior observed here is consistent with the preponderance of centrifugal motions in the visual periphery, providing new evidence for a link between environmental statistics and perceptual assumptions. Additionally, this finding adds to the large number of differences between central and peripheral motion perception (Brandt et al., 1973; Baker and Braddick, 1985; Lappin et al., 2009; Tadin et al., 2012).

How does the recovered prior correspond to naturally experienced motion signals? If we make a gross simplification and assume that the visual periphery is only exposed to the expanding optic follow, then a centrifugal prior peaking at $8 \%$ translates to $0.65 \mathrm{~km} / \mathrm{h}$ walking speed at our display conditions ( $83 \mathrm{~cm}$ viewing distance; $40^{\circ}$ eccentricity). This is considerably less than the typical walking speed $(\sim 5 \mathrm{~km} / \mathrm{h})$. Of course, our visual experience is not restricted to the expanding optic flow. These other forms of visual input would likely attenuate centrifugal biasing of motion perception. For example, peripheral motion inputs due to smooth pursuit eye movements (SPEMs) are unlikely to exhibit a centrifugal bias (Kowler, 2011). SPEMs are a potent source of fast peripheral motions $(<30 \% \mathrm{~s})$ and might partially account for the centripetal component of recovered nonparametric priors (Fig. 4). However, whether the observed centripetal component is indeed due to SPEMs is speculative. Given the close coupling of oculomotor and motion processing (Kowler, 2011), it is pos- sible that motion perception during SPEMs might be associated with a distinct set of prior assumptions. The centripetal component might also reflect our experience with the contracting optic flow (e.g., while swaying away from a computer monitor). Finally, other peripheral visual inputs are unlikely to exhibit strong centrifugal biases and, thus, likely attenuate the effects of the optic flow. In contrast, we also experience very fast self-motions such as driving (although all participants tested in this study have very short commutes). In sum, while it is safe to assume that our experience with peripheral motions has a strong centrifugal bias, it is difficult to link the observed results to real life experiences. An additional complication is that the velocity distribution of visual motion inputs also depends on stimulus eccentricity and viewing distance. Stimuli that are closer to fovea will have weaker centrifugal biases, while more distant stimuli will tend to have slower retinal velocities. Thus, it will be important for future work to also consider these two parameters.

In the Bayesian framework, the effects of the prior become progressively more apparent as the stimulus uncertainty increases (Körding and Wolpert, 2004), simply reflecting the fact that when incoming sensory signals are unreliable, the internal default estimate (i.e., the prior) becomes a better choice. Here, we degraded the reliability of motion direction by decreasing stimulus duration. As the stimulus duration decreases, its spread in the temporal frequency space increases, decreasing the reliability of velocity estimates (Fig. 1C; Derrington and Goddard, 1989). This occurs for both moving and stationary stimuli, with the only difference being that the motion energy of stationary stimuli (of finite duration) is balanced between opposing motion directions (i.e., centered at $0 \mathrm{~Hz}$ ). Thus, changes in stimulus duration provide a simple way to manipulate the uncertainty of motion signals. Stimulus contrast is often used to accomplish the same aim (Stocker and Simoncelli, 2006). However, for our rather large and brief grating stimuli, changes in stimulus contrast have nonlinear, interactive effects on motion discriminations-effects stemming from contrast-dependent changes in spatial suppression (Tadin et al., 2003). Moreover, spatial suppression strength also interacts with stimulus speed (Lappin et al., 2009), providing an additional complication. For these reasons, we chose to use stimulus duration to manipulate stimulus uncertainty, estimating Bayesian likelihood from empirically observed velocity JNDs (Fig. 3A). Note that because of a close relationship between measured JNDs and stimulus spread in frequency space (Fig. 3B), stimulus description in frequency space could be used to directly estimate Bayesian likelihood.

The centrifugal prior reported here is consistent with previous reports of directional anisotropies in the visual periphery, providing a possible computational explanation for those 
earlier findings. For random-dot stimuli, motion detection and discrimination thresholds are lower for centrifugal than for centripetal motion directions (Ball and Sekuler, 1980). The same results were found for second-order stimuli (Smith et al., 1994). Counterphase gratings (stimuli composed of two superimposed gratings moving in opposite directions) appear to move centrifugally when presented in the periphery, indicating that the centrifugal motion component dominates over the centripetal component (Georgeson and Harris, 1978). Consistent with this finding, centrifugal motions yield stronger MEG signals than centripetal motions (Holliday and Meese, 2008). This centrifugal advantage is likely a key factor in the so-called "radial bias," where stronger BOLD responses are found in radial, rather than tangential, motions (Raemaekers et al., 2009). In sum, these studies are broadly consistent with a centrifugal bias in motion perception. Our results directly demonstrate that this bias is quite strong, affecting both moving and stationary stimuli, and show that it can be explained by a centrifugal Bayesian prior.

What neural mechanism might underlie the observed centrifugal bias in the visual periphery? For orientation discriminations, observed perceptual biases can be explained by a nonuniform distribution of orientation preferences (Girshick et al., 2011), such as that found for V1 neurons (De Valois et al., 1982). Similar to orientation tuning, tuning preferences of motion selective neurons are not uniformly distributed. In cortical area MT, a key motion processing region (Born and Bradley, 2005), neurons with peripheral receptive fields tend to have centrifugal preferred directions (Albright, 1989). Similar biases are found in V1 (Holliday et al., 2008). Such tuning anisotropies can bias neural responses to moving stimuli, especially if the distribution of stimulus motion directions is broad. For example, consider a brief stimulus moving toward the fovea. Because of its broad temporal frequency spectrum, this motion will stimulate detectors tuned to both centripetal and centrifugal motion directions. As the physical motion direction is centripetal, those units will have stronger inputs. However, if neural processing is biased in favor of centrifugally tuned units, the resultant estimate may differ from the physical motion direction. This same scenario also applies to stationary stimuli, which for subsecond durations have nontrivial spreads in the temporal frequency spectrum. In fact, such brief stationary stimuli provide an elegant way to reveal motion perception biases because they are balanced in terms of directional motion energy. Thus, even a small imbalance in motion decoding might result in perceived motion direction. Indeed, even the longest stimulus that we used ( $\sigma=240 \mathrm{~ms}$, yielding about $1 \mathrm{~s}$ total duration) tended to be perceived as moving away from fovea (Fig. 2). This result, however, does not indicate that we should routinely perceive peripherally presented stationary objects as moving. Nulling speeds for the 240 ms stimulus were only $0.2^{\circ}$ s (Fig. $2 B$ ), which, during natural vision, is unlikely to evoke a compelling or even noticeable sensation of motion in the visual periphery. One exception would be briefly presented stationary stimuli, such as a light flash. Based on our results, we speculate that even in natural environments such stimuli would be perceived as moving centrifugally. To speculate even further, we suspect that illusory motion of stationary flashes in visual periphery would contribute to the attentional salience of such stimuli.

\section{References}

Adams WJ, Graf EW, Ernst MO (2004) Experience can change the 'lightfrom-above' prior. Nat Neurosci 7:1057-1058. CrossRef Medline

Albright TD (1989) Centrifugal directional bias in the middle temporal visual area (MT) of the macaque. Vis Neurosci 2:177-188. CrossRef Medline

Baker CL Jr, Braddick OJ (1985) Eccentricity-dependent scaling of the limits for short-range apparent motion perception. Vis Res 25:803-812. CrossRef Medline

Ball K, Sekuler R (1980) Human vision favors centrifugal motion. Perception 9:317-325. CrossRef Medline

Born RT, Bradley DC (2005) Structure and function of visual area MT. Annu Rev Neurosci 28:157-189. CrossRef Medline

Brainard DH (1997) The psychophysics toolbox. Spat Vis 10:433-436. CrossRef Medline

Brandt T, Dichgans J, Koenig E (1973) Differential effects of central versus peripheral vision on egocentric and exocentric motion perception. Exp Brain Res 16:476-491. CrossRef Medline

Chalk M, Seitz AR, Series P (2010) Rapidly learned stimulus expectations alter perception of motion. J Vis 10(8):2. CrossRef Medline

Dakin SC, Mareschal I, Bex PJ (2005) An oblique effect for local motion: psychophysics and natural movie statistics. J Vis 5(10):878-887. CrossRef Medline

De Bruyn B, Orban GA (1988) Human velocity and direction discrimination measured with random dot patterns. Vision Res 28:1323-1335. CrossRef Medline

Derrington AM, Goddard PA (1989) Failure of motion discrimination at high contrasts: evidence for saturation. Vision Res 29:1767-1776. CrossRef Medline

De Valois RL, Yund EW, Hepler N (1982) The orientation and direction selectivity of cells in macaque visual cortex. Vision Res 22:531-544. CrossRef Medline

Ernst MO, Banks MS (2002) Humans integrate visual and haptic information in a statistically optimal fashion. Nature 415:429-433. CrossRef Medline

Geisler WS (2008) Visual perception and the statistical properties of natural scenes. Annu Rev Psychol 59:167-192. CrossRef Medline

Georgeson MA, Harris MG (1978) Apparent foveofugal drift of counterphase gratings. Perception 7:527-536. CrossRef Medline

Girshick AR, Landy MS, Simoncelli EP (2011) Cardinal rules: visual orientation perception reflects knowledge of environmental statistics. Nat Neurosci 14:926-932. CrossRef Medline

Hedges JH, Stocker AA, Simoncelli EP (2011) Optimal inference explains the perceptual coherence of visual motion stimuli. J Vis. 11(6) pii:14. CrossRef Medline

Holliday IE, Meese TS (2008) Optic flow in human vision: MEG reveals a foveo-fugal bias in V1, specialization for spiral space in hMSTs, and global motion sensitivity in the IPS. J Vis. 8(10):17 1-24. ${ }^{10.1167 / 8.10 .17 ~ C r o s s R e f ~}$

Hürlimann F, Kiper DC, Carandini M (2002) Testing the Bayesian model of perceived speed. Vision Res 42:2253-2257. CrossRef Medline

Kersten D (1994) Visual perception as Bayesian 'inference'. Perception 23:6.

Kersten D, Knill DC, Mamassian P, Bülthoff I (1996) Illusory motion from shadows. Nature 379:31. CrossRef Medline

Kersten D, Mamassian P, Yuille A (2004) Object perception as Bayesian inference. Annu Rev Psychol 55:271-304. CrossRef Medline

Knill DC, Pouget A (2004) The Bayesian brain: the role of uncertainty in neural coding and computation. Trends Neurosci 27:712-719. CrossRef Medline

Körding KP, Wolpert DM (2004) Bayesian integration in sensorimotor learning. Nature 427:244-247. CrossRef Medline

Kowler E (2011) Eye movements: the past 25 years. Vision Res 51:14571483. CrossRef Medline

Lappin JS, Tadin D, Nyquist JB, Corn AL (2009) Spatial and temporal limits of motion perception across variations in speed, eccentricity, and low vision. J Vis 9(1):30 1-14. CrossRef Medline

Leonard T (1978) Density estimation, stochastic processes and prior information. J R Statist Soc B 40:113-146.

Levitt H (1971) Transformed up-down methods in psychoacoustics. J Acoust Soc Am 49 [Suppl 2]:467+. CrossRef Medline

McKee SP (1981) A local mechanism for differential velocity detection. Vision Res 21:491-500. CrossRef Medline 
Orban GA, de Wolf J, Maes H (1984) Factors influencing velocity coding in the human visual system. Vision Res 24:33-39. CrossRef Medline

Pelli DG (1997) The VideoToolbox software for visual psychophysics: transforming numbers into movies. Spat Vis 10:437-442. CrossRef Medline

Raemaekers M, Lankheet MJ, Moorman S, Kourtzi Z, van Wezel RJ (2009) Directional anisotropy of motion responses in retinotopic cortex. Hum Brain Mapp 30:3970-3980. CrossRef Medline

Seydell A, Knill DC, Trommershauser J (2010) Adapting internal statistical models for interpreting visual cues to depth. J Vis 10(4):1 1-27. CrossRef Medline

Simoncelli EP, Olshausen BA (2001) Natural image statistics and neural representation. Annu Rev Neurosci 24:1193-1216. CrossRef Medline

Smith AT, Hess RF, Baker CL Jr (1994) Direction identification thresholds for second-order motion in central and peripheral vision. J Opt Soc Am A Opt Image Sci Vis 11:506-514. CrossRef Medline

Sotiropoulos G, Seitz AR, Seriès P (2011) Changing expectations about speed alters perceived motion direction. Curr Biol 21:R883-R884. CrossRef Medline
Stocker AA, Simoncelli EP (2006) Noise characteristics and prior expectations in human visual speed perception. Nat Neurosci 9:578-585. CrossRef Medline

Tadin D, Lappin JS, Gilroy LA, Blake R (2003) Perceptual consequences of centre-surround antagonism in visual motion processing. Nature 424:312-315. CrossRef Medline

Tadin D, Nyquist JB, Lusk KE, Corn AL, Lappin JS (2012) Peripheral vision of youths with low vision: motion perception, crowding, and visual search. Invest Ophthalmol Vis Sci 53:5860-5868. CrossRef Medline

Thompson P (1982) Perceived rate of movement depends on contrast. Vision Res 22:377-380. CrossRef Medline

Warren WH Jr, Kay BA, Zosh WD, Duchon AP, Sahuc S (2001) Optic flow is used to control human walking. Nat Neurosci 4:213-216. CrossRef Medline

Weiss Y, Simoncelli EP, Adelson EH (2002) Motion illusions as optimal percepts. Nat Neurosci 5:598-604. CrossRef Medline

Wichmann FA, Hill NJ (2001) The psychometric function: I. fitting, sampling, and goodness of fit. Percept Psychophys 63:12931313. CrossRef Medline 